\title{
GRADE AND RECOVERY PREDICTION FOR EDDY CURRENT SEPARATION PROCESSES
}

\author{
P.C. REM*, E.M. BEUNDER and W. KUILMAN \\ Faculty of Applied Earth Sciences, Delft University of Technology, \\ Mijnbouwstraat 120, 2628 RX Delft, The Netherlands
}

(Received 28 March 1998; Accepted 5 May 1998)

Grade and recovery of eddy current separation can be estimated on the basis of trajectory simulations for particles of simple shapes. In order to do so, the feed is characterized in terms of a small set of test-particles, each test-particle representing a fraction of the feed of a given size, shape and material. In this paper, the grade and recovery curve predicted for a sample from the 6-16 mm non-ferrous fraction of car scrap is compared with experimental data. The results indicate that it may be possible to automatically control eddy current separators on the basis of such predictions.

Keywords: Eddy current; Modelling; Grade and recovery; Non-ferrous

\section{O INTRODUCTION}

Eddy current separation is an effective way of recovering non-ferrous metals from streams of industrial or municipal waste [1]. The separation is brought about by inducing eddy currents inside the conductive particles of the stream. These currents lend a transient magnetic moment to the particles which is used to propel them in a gradient magnetic field [2]. Among the many design concepts for separators that have been tried, the rotary drum is the most widely used type of eddy current separator today (see Fig. 1). The active part of this machine is a fast spinning drum, with a surface consisting of rows of magnets of alternating

\footnotetext{
* Corresponding author.
} 


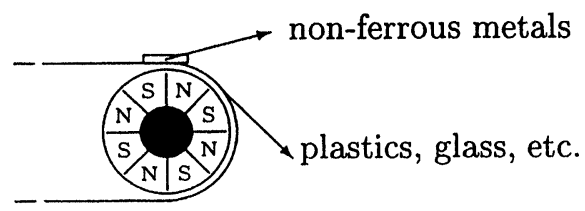

FIGURE 1 An eddy current separator consisting of a drum covered with magnets that are oriented alternately $N-S$ and $S-N$. The fluctuating field of the spinning drum induces eddy currents in electrically conductive particles moving close to the drum. These particles are then expelled from the feed stream.

polarity. A conveyor belt takes the feed over the drum and the conductive particles are ejected from the mainstream. The trajectory of these particles is generally determined by a combination of the electromotive force, gravity, and the forces of friction with the conveyor belt and the air.

At present, none of the published models for eddy current processes can claim complete generality. Most of the theory developed before the nineties, in particular the work by Schlömann $[3,4]$ and van der Valk et al. [5-7], is dealing with the limit of small particles or low frequencies, and ignores the effect of particle rotation. In the early nineties, work was done on larger particles by Fletcher et al. [8-10], starting from first principles. The results of this model show a fair correspondence with experimental results. However, both particle and field geometry were different from what is common in practical applications. Recently, Meier Staude and Mersmann [11] simulated a rotary drum including the conveyor belt. Their model ignores the radial component of the magnetic force, however, and has been demonstrated only for special particle shapes.

The model used for the simulations in this paper was developed by treating the particles as magnetic dipoles [12-14]. This kind of model is limited to small and medium-sized particles but it can deal with any kind of field geometry. So far, it has been applied to rather basic particle shapes like spheres and cylinders, but there is no fundamental reason why it should not be developed for more complex shapes. The basic theory of the electromagnetic forces and the model for the mechanical interactions of the particles with the belt are briefly discussed in the next section. The remainder of the paper describes the prediction of grade and recovery on the basis of trajectory simulations. The procedure of 
feed characterization is explained and the predicted grade and recovery curves are compared with experimental data for an example case.

\section{THE DIPOLE MODEL}

The force $\mathbf{F}$ and torque $\mathbf{T}$ exerted on a particle by an external magnetic field $\mathbf{B}^{a}$ can be expressed in terms of the field gradient and the particle magnetization [15]. If the field gradient $\nabla \mathbf{B}^{a}$ is relatively constant within the volume of the particle, the expressions become very simple:

$$
\begin{gathered}
\mathbf{F}=\mathbf{M} \cdot \nabla \mathbf{B}^{a} \\
=M_{x} \nabla B_{x}^{a}+M_{y} \nabla B_{y}^{a}+M_{z} \nabla B_{z}^{a}, \\
\quad \mathbf{T}=\mathbf{M} \times \mathbf{B}^{a} .
\end{gathered}
$$

In these formulas, $\mathbf{M}$ is the magnetic (dipole) moment of the particle. For eddy current separation, the above approximation is sufficiently accurate if particles are smaller than about one third of the wavelength of the magnetic field (or equivalently, a third of the width of a pair of magnetic poles). This means that for rotary drum machines the model is limited to particles with diameters less than $30-50 \mathrm{~mm}$.

It was shown in [14] that the dynamics of the particle magnetic moment $\mathbf{M}$, as observed by the particle in its own frame of reference, can be closely approximated by a linear, first order differential equation:

$$
\frac{\mathrm{d}}{\mathrm{d} t} \mathbf{M}=-\frac{\mathbf{C}}{\mu_{0} \sigma R^{2}} \cdot \mathbf{M}-\frac{V}{\mu_{0}} \mathbf{D} \cdot \frac{\mathrm{d}}{\mathrm{d} t} \mathbf{B}^{a} .
$$

In this formula, $\mathbf{B}^{a}$ is the magnetic field as observed in the particle's frame of reference, $\sigma$ is the electrical conductivity of the particle and $\mu_{0}=4 \pi \cdot 10^{-7} \mathrm{Tm} / \mathrm{A}$ is the magnetic permeability of vacuum. $R$ defines the size of the particle and $V$ is its volume. The tensors $\mathbf{C}$ and $\mathbf{D}$ are dimensionless and depend only on the shape of the particle. For example, for a cylindrical particle, oriented with its long axis along the $z$-axis of its frame of reference:

$$
\mathbf{C}=6\left(\begin{array}{lll}
1 & 0 & 0 \\
0 & 1 & 0 \\
0 & 0 & 1
\end{array}\right),
$$


and

$$
\mathbf{D}=\frac{3}{4}\left(\begin{array}{lll}
2 & 0 & 0 \\
0 & 2 & 0 \\
0 & 0 & 1
\end{array}\right)
$$

In the absence of other forces, Eqs. (1)-(3), in combination with Newton's laws of motion, can be integrated for the trajectory of the particle [16].

\subsection{Contact Forces}

The most important force besides the electromotive force is the mechanical interaction of the particles with the conveyor belt. Interparticle forces may also play a role, especially at higher throughputs, but these forces were not taken into account. In order to avoid multiple points of contact between the particle and the belt, the shape of the particles was, somewhat arbitrarily, represented by the largest internal ellipsoid. The dynamical states of the system were confined to three modes: roll, slide and fly.

The transition between rolling and sliding was defined by the standard criterion for the friction force in terms of the support force $F^{\perp}$ and the coefficient of static friction $f^{\mathrm{s}}$ :

$$
\left|\mathbf{F}^{\text {fric }}\right|>f^{\mathrm{s}} F^{\perp}
$$

For the slide mode a modification of Coulomb's law of dry friction was used to guarantee a smooth transition back to rolling:

$$
\mathbf{F}^{\text {fric }}=f^{\mathrm{d}} F^{\perp} \frac{\Delta \mathbf{u}}{|\Delta \mathbf{u}|+\epsilon} .
$$

Here, $f^{\mathrm{d}}$ is the dynamic friction factor, $\Delta \mathbf{u}$ is the differential velocity between the belt and the particle at their point of contact, and $\epsilon$ is a very small velocity constant. The transition from sliding to flying was defined by the criterion $F^{\perp}<0$. Occasional collisions of particles with the belt were simulated by the rigid body collision model of Keller [17,18], with restitution coefficients of about 0.2 . 


\subsection{Aerodynamic Forces}

Aerodynamic forces of drag, and also of lift and torque (for fast spinning particles) are relatively unimportant compared to the electromotive and mechanical forces. Nevertheless, their effects can be traced experimentally [14]. In the present simulations basic formulas for the turbulent limit were applied for all cases.

\section{PREDICTION OF GRADE AND RECOVERY}

Suppose that a mass $F$ of feed material is processed into a mass $P$ of product and a tailing. Furthermore, suppose that $F_{m}$ and $P_{m}$ are the mass fractions of a material (or class of materials) $m$ in the feed and product, respectively. Then the grade $G_{m}$ and the recovery $R_{m}$ of the material $m$ in the product are defined as usual:

$$
\begin{aligned}
G_{m} & =P_{m} / P, \\
R_{m} & =P_{m} / F_{m} .
\end{aligned}
$$

A grade-recovery diagram can be made by plotting the grade versus the recovery for a number of operating conditions of the eddy current separator. The interesting question is whether it is possible to predict the outer envelop of the points in the grade-recovery diagram: the grade-recovery curve.

The first step in making the prediction is to represent the actual feed by a small set of test-particles, i.e., model particles with properties similar to the particles of some fraction of the feed. Then the trajectories of these test-particles are computed for a number of belt speeds and drum speeds of the separator (cf. Fig. 2). From the resulting data, the predicted grade and recovery can be computed for each combination of belt speed, drum speed and position of the splitter. Finally, the grade-recovery curve is obtained by rejecting all points from the graderecovery diagram that are inferior to some other point in both grade and recovery.

\subsection{Feed Characterization}

The feed of an eddy current process usually covers a wide range of particle shapes, sizes and materials. At present, it seems unpractical to 


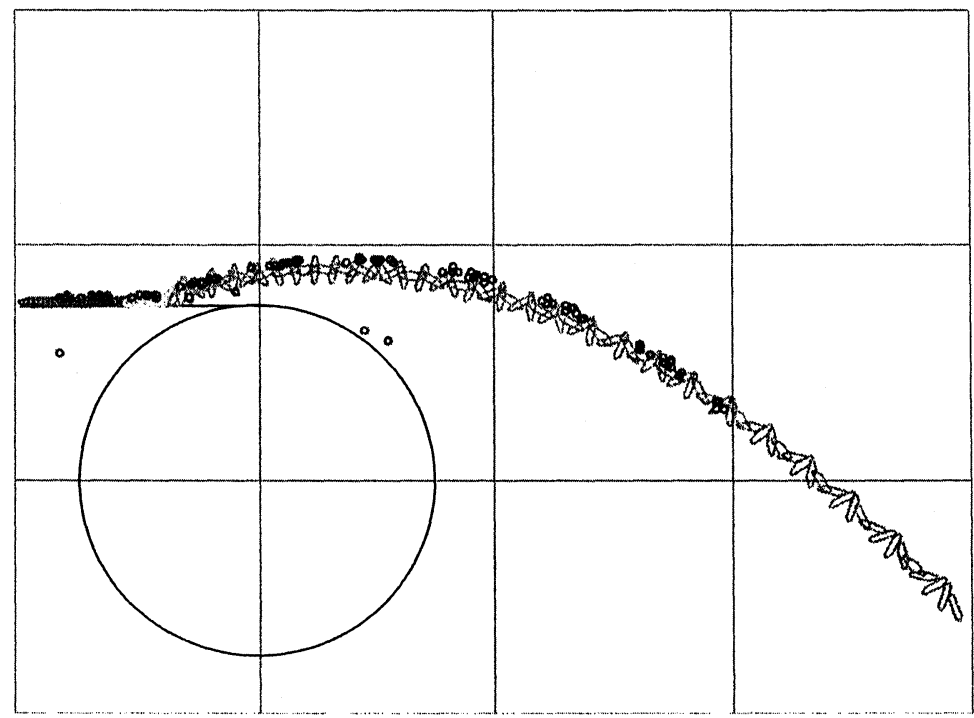

FIGURE 2 Simulated trajectory for a cylindrical aluminium particle (diameter $8 \mathrm{~mm}$, length $24 \mathrm{~mm}$ ), processed on a BM 29.701/18 [19] eddy current separator at a belt speed of $1 \mathrm{~m} / \mathrm{s}$ and drum speed of $50 \mathrm{rps}$. The open circles are experimental data for the same conditions.

try to accurately model a large number of these particles and compute their trajectories. Instead, a representative sample of the feed is split into several fractions, each fraction consisting of particles of the same material or class of materials and of roughly similar shape. Each fraction is then represented by three or four test-particles of the same material and idealized shape, but of different sizes. Finally, a mass of feed is assigned to each test-particle that reflects the distribution of mass over the materials, shapes and sizes of the particles in the sample.

In our experiment, we selected a sample from the $6-16 \mathrm{~mm}$ of the non-ferrous fraction of car scrap (see Fig. 3). All separation experiments were done with this sample, which was carefully reconstructed after each run. For the purpose of characterization, the sample was first divided into four shape categories: flat particles, globular particles and two kinds of rod-shaped particles (all-metal and insulated wire). The flat particles were represented by circular disks, the globular particles by spheres and the rod-shaped particles by cylinders. These categories were split again, into subfractions of aluminium, copper, brass, stainless 


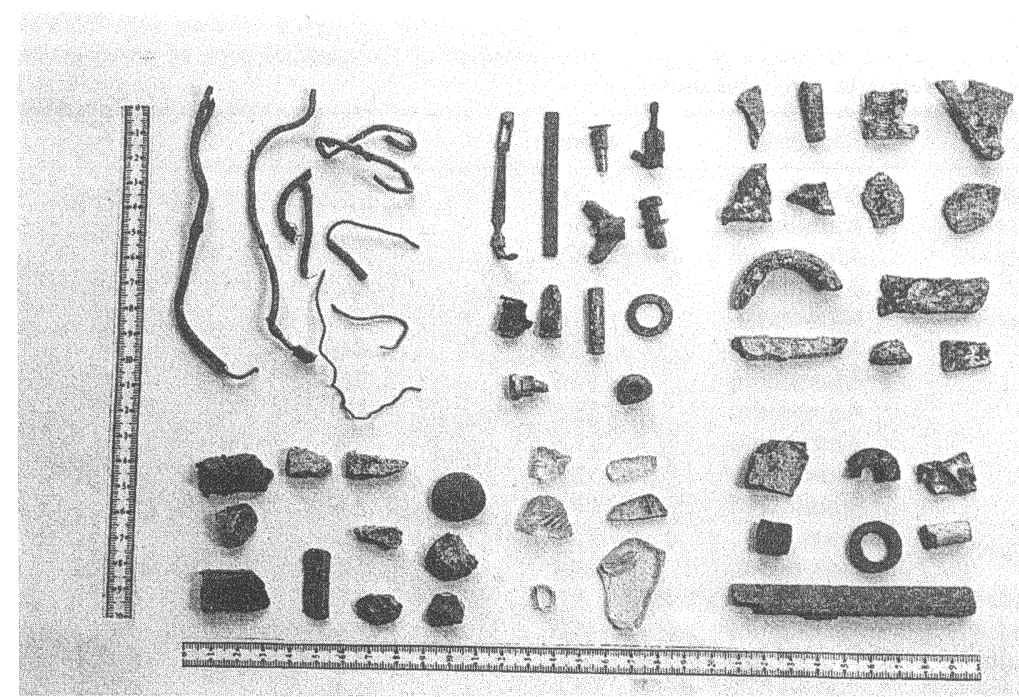

FIGURE 3 The $6-16 \mathrm{~mm}$ non-ferrous source fraction of car scrap from which the sample for the experiments were taken.

steel and fully non-metallic particles. Finally, some of the larger sets of particles were subdivided according to size. For flat particles the largest diameter was taken as a basis for size classification. The final list of testparticles is shown in Table $\mathrm{I}$.

\subsection{Simulations}

The list of test-particles was fed to simulation software based on the dipole model discussed in the previous section in order to compute the particle trajectories for five different belt speeds ranging from 1 to $2 \mathrm{~m} / \mathrm{s}$ and drum speeds of 30,40 and 50 rps on a BM 29.701/18 eddy current separator [19]. For a given belt speed and drum speed, the splitter position was varied along a line extending radially from the center of the rotor (see Fig. 4). Five lines of splitter positions were selected, ranging from horizontal to almost vertical $\left(\phi=0^{\circ}, 20^{\circ}, 40^{\circ}, 60^{\circ}, 80^{\circ}\right)$, and for each line, the radii of intersection with the particle trajectories were computed. The splitter position $(x, y)$ was then stepped up from the minimum radius to the maximum radius in ten steps and for each position the theoretical grade and recovery was determined, both for 
TABLE I List of test-particles representing a sample of the $6-16 \mathrm{~mm}$ non-ferrous fraction of car scrap shown in Fig. 3. The diameter of the plastics part of wires given in the table refers to the outer diameter

\begin{tabular}{llccc}
\hline Shape & Material & $d(\mathrm{~mm})$ & lor $h(\mathrm{~mm})$ & Mass $(\mathrm{g})$ \\
\hline Sphere & Aluminium & 5.0 & & 0.6 \\
Sphere & Aluminium & 6.3 & & 2.2 \\
Sphere & Aluminium & 8.7 & & 4.8 \\
Sphere & Aluminium & 12.5 & & 11.5 \\
Sphere & Non-metal & 15.0 & & 222.5 \\
Disk & Aluminium & 12.5 & 4.0 & 7.0 \\
Disk & Aluminium & 17.5 & 5.0 & 17.1 \\
Disk & Aluminium & 22.5 & 5.0 & 10.7 \\
Disk & Aluminium & 27.5 & 6.0 & 12.3 \\
Disk & Aluminium & 32.5 & 4.0 & 10.9 \\
Disk & Stainless & 17.5 & 2.0 & 1.2 \\
Disk & Brass & 12.5 & 2.0 & 1.5 \\
Disk & Non-metal & 20.0 & 5.0 & 35.0 \\
Cylinder & Aluminium & 5.0 & 10.0 & 0.7 \\
Cylinder & Aluminium & 7.0 & 15.0 & 5.5 \\
Cylinder & Aluminium & 9.0 & 25.0 & 6.8 \\
Cylinder & Copper & 1.5 & 32.5 & 0.9 \\
Cylinder & Copper & 0.7 & 35.0 & 0.05 \\
Cylinder & Brass & 10.0 & 17.5 & 4.9 \\
Cylinder & Brass & 8.0 & 79.0 & 29.7 \\
Cylinder & Brass & 10.0 & 25.0 & 9.6 \\
Cylinder & Stainless & 10.0 & 21.0 & 10.3 \\
Cylinder & Non-metal & 10.0 & 35.0 & 29.0 \\
Wire & Copper & 2.5 & 35.0 & \\
& Plastics & 3.0 & & 1.5 \\
Wire & Copper & 1.5 & 22.0 & \\
& Plastics & 2.0 & & 0.6 \\
Wire & Copper & 1.7 & 53.0 & 0.6 \\
& Plastics & 2.5 & & \\
\hline
\end{tabular}

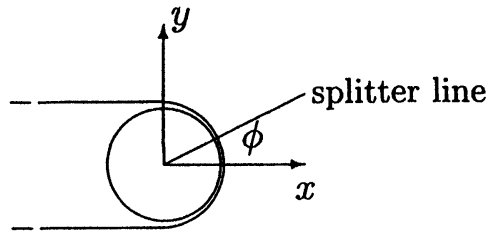

FIGURE 4 Splitter position is varied along lines in the $x-y$ plane.

aluminium and for the non-ferrous materials as a whole. The resulting grade-recovery curve for aluminium is presented in Table II.

The table shows that the prediction favors a combination of high drum speed and a relatively high belt speed, which is consistent with the 
TABLE II Prediction of optimal settings and grade and recovery for aluminium in the product

\begin{tabular}{lccccc}
\hline Drum $(\mathrm{rps})$ & Belt $(\mathrm{m} / \mathrm{s})$ & $x(\mathrm{~m})$ & $y(\mathrm{~m})$ & $G_{\mathrm{Al}}$ & $R_{\mathrm{Al}}$ \\
\hline 50.00 & 1.75 & 0.352 & 0.000 & 65.81 & 99.72 \\
50.00 & 1.75 & 0.247 & 0.090 & 66.51 & 98.71 \\
50.00 & 2.00 & 0.284 & 0.103 & 70.34 & 95.24 \\
50.00 & 1.75 & 0.160 & 0.134 & 71.49 & 94.90 \\
50.00 & 1.75 & 0.285 & 0.104 & 72.46 & 93.50 \\
50.00 & 1.75 & 0.522 & 0.000 & 76.51 & 92.21 \\
50.00 & 2.00 & 0.329 & 0.120 & 89.04 & 87.07 \\
50.00 & 1.50 & 0.520 & 0.000 & 92.23 & 85.51 \\
50.00 & 1.75 & 0.578 & 0.000 & 97.10 & 85.45 \\
50.00 & 2.00 & 0.374 & 0.136 & 98.99 & 82.62 \\
50.00 & 1.75 & 0.635 & 0.000 & 100.00 & 79.13 \\
\hline
\end{tabular}

TABLE III Experimental results for the grade and recovery of aluminium and total non-ferrous in the product. The splitter position was fixed at $(x, y)=(0.187,0.069) \mathrm{m}$

\begin{tabular}{lccccr}
\hline Drum $(\mathrm{rps})$ & Belt $(\mathrm{m} / \mathrm{s})$ & $G_{\mathrm{Al}}$ & $R_{\mathrm{Al}}$ & $G_{\mathrm{nf}}$ & $R_{\mathrm{nf}}$ \\
\hline 30 & 1.68 & 22.56 & 100.0 & 35.34 & 100.00 \\
30 & 1.51 & 24.68 & 98.65 & 38.11 & 97.21 \\
30 & 1.33 & 24.74 & 98.88 & 80.51 & 94.13 \\
30 & 1.16 & 63.29 & 93.27 & 96.19 & 90.47 \\
40 & 1.68 & 22.84 & 100.0 & 35.79 & 100.00 \\
40 & 1.51 & 24.43 & 98.77 & 38.40 & 99.07 \\
40 & 1.33 & 52.81 & 99.10 & 80.20 & 96.06 \\
40 & 1.16 & 62.12 & 98.09 & 94.46 & 95.20 \\
50 & 1.68 & 22.47 & 99.89 & 35.17 & 99.79 \\
50 & 1.51 & 24.34 & 99.89 & 38.10 & 99.79 \\
50 & 1.33 & 53.90 & 97.76 & 83.35 & 96.49 \\
50 & 1.16 & 59.53 & 93.6 & 91.72 & 92.05 \\
\hline
\end{tabular}

small size of the material [14]. The best splitter position is generally low, which is also the experience from the field.

\subsection{Experiments}

The sample used in the characterization was also used as the feed for a number of separation experiments on a BM 29.701/18 eddy current separator. The grade and recovery both for aluminium and total nonferrous were determined at several drum speeds ( 30,40 and $50 \mathrm{rps})$ and belt speeds $(1.16,1.33,1.51$ and $1.68 \mathrm{~m} / \mathrm{s})$. The splitter position was fixed at $(x, y)=(0.187,0.069) \mathrm{m}$ in all runs (see Table III). 


\subsection{Comparison of Results}

Figure 5 shows the grade-recovery diagram for aluminium, with the experimental datapoints indicated by diamonds. The solid line represents the simulated grade-recovery curve. Note that the simulation shows a drop in recovery just above $60 \%$ grade, in agreement with experimental data. As a whole, the simulation is somewhat more optimistic than the experimental datapoints. In order to check whether this was due to the wider range of splitter positions covered by the simulation, the simulation results for splitter positions close to the experimental position were added to the diagram as separate points $(+)$. Comparison of the data shows that, indeed, part of the difference can be contributed to this effect, but the simulation remains slightly more optimistic. A similar result is found, comparing the simulated and experimental results for the non-ferrous product in Fig. 6, with the exception that for low grades, there are actually a few experimental datapoints beyond the simulated grade-recovery curve. In general, however, the results for this case show that the computed curve deviates only a few percent in grade and recovery from the experiments.

\subsection{Process Control}

The present technology for predicting the grade and recovery may be combined with a sensor that automatically characterizes the feed

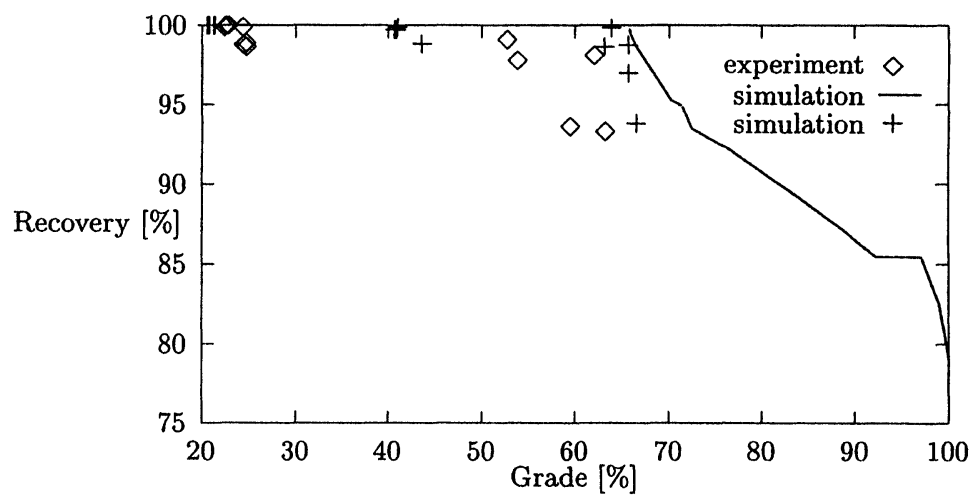

FIGURE 5 Simulated grade-recovery curve for the aluminium product versus data from experiments. The points marked + are simulated points for splitter positions closest to that of the experiments. 


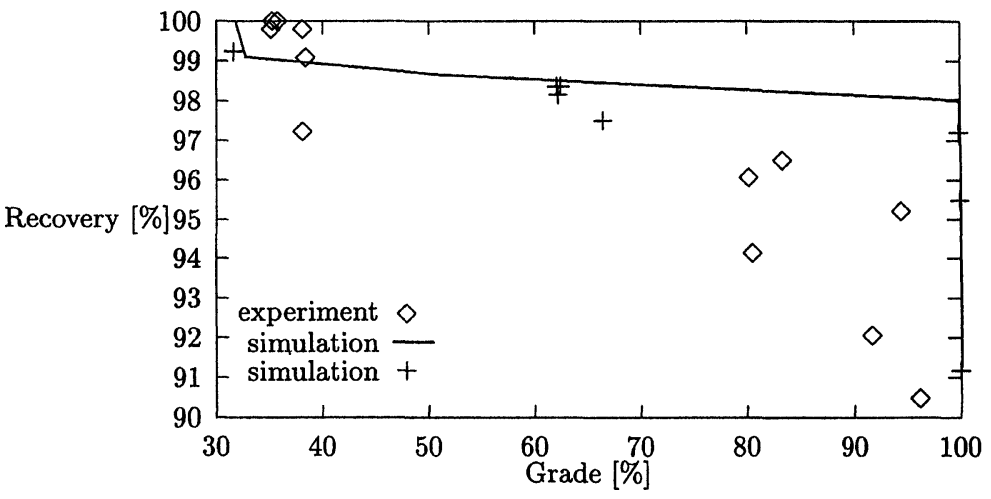

FIGURE 6 Simulated grade-recovery curve for the non-ferrous product versus data from experiments. The points marked + are simulated points for splitter positions closest to that of the experiments.

material in order to arrive at a system for controlling the eddy current separation process. A prototype of such a sensor is currently being built at our laboratory. The sensor estimates the size, shape and material class of about $10 \%$ of the particles that are being processed by the separator. On the basis of the statistics that are generated, the separator settings can be adjusted automatically, say, once every few minutes, to improve the separation results.

\section{CONCLUSIONS}

The first results of simulating grade and recovery for car scrap by characterizing the feed in terms of model particles and simulating their trajectories show a fair agreement with experimental results. Simulations of this kind may help in the selection of process schemes and the design of eddy current separators. Another potential application of this kind of analysis is on-line process control, given a sensor that automatically characterizes the feed material.

\section{References}

[1] Dalmijn, W.L., Practical application of eddy currents in the scrap recycling, in Proc. Second Int. Symposium Recycling of Metals and Engineering Materials, The Minerals, Metals \& Materials Society, Pennsylvania, USA (1990). 
[2] Edison, T.A., U.S. Patent 400,317 (1889).

[3] Schlömann, E., Separation of nonmagnetic materials from solid waste by permanent magnets. I. Theory, J. Appl. Phys. 46(11) (1975) 5012.

[4] Schlömann, E., Separation of nonmagnetic metals from solid waste by permanent magnets. II. Experiments on circular disks, J. Appl. Phys. 46(11) (1975) 5022.

[5] van der Valk, H.J.L., Dalmijn, W.L. and Duyvesteyn, W.P.C., Erzmetall 41 (1988) 266.

[6] van der Valk, H.J.L., Braam, B.C. and Dalmijn, W.L., Eddy-current separation by permanent magnets Part I: Theory, Resources, and Conservation 12 (1986) 233.

[7] Braam, B.C., van der Valk, H.J.L. and Dalmijn, W.L., Eddy-current separation by permanent magnets Part II: Rotating disc separators, Resources, Conservation and Recycling 1 (1988) 3.

[8] Fletcher, D., Gerber, R., Lawson, P. and Boehm, J., Eddy current separation of non-ferrous conductors and non-conductors: theory and initial experiments, IEEE Trans. Mag. 27(6) (1991) 5375.

[9] Fletcher, D., Gerber, R., Tarrant, L. and Reid, T., Experimental validation and generalized theory of a single boundary eddy-current separator model, IEEE Trans. Mag. 28(5) (1992) 2415.

[10] Fletcher, D. and Gerber, R., Electromagnetic separation: the prediction and measurement of conductor separability, IEEE Trans. Mag. 29(6) (1993) 3255.

[11] Meier-Staude, R. and Mersmann, A., Analytische Modellierung der Partikeltrajektorien bei der Wirbelstromscheidung, Schüttgut 3 (1997) 307.

[12] Leest, P.A., Rem, P.C. and Dalmijn, W.L., Analytical approach for custom designing of eddy current separators, in Proc. XLVI. Berg- und Hüttenmännischer Tag (1995) Freiberg.

[13] Rem, P.C., Leest, P.A. and van der Akker, A.J., A model for eddy current separation, Int. J. Min. Proc. 49 (1997) 193.

[14] Rem, P.C., Beunder, E.M. and van der Akker, A.J., Simulation of eddy current separators, submitted to IEEE Trans. Mag. 34(4) (1998) 2280.

[15] For example see Landau, L.D. and Lifshitz, E.M., Electrodynamics of Continuous Media, Pergamon Press, London (1963) p. 143.

[16] Brenan, K.E., Campbell, S.L. and Petzold, L.R., Numerical solution of initial value problems, in Differential-Algebraic Equations, Elsevier, New York (1989).

[17] Keller, J.B., Impact with friction, J. Appl. Mech. 53 (1986) 1.

[18] Wang, Y. and Mason, M.T., Two-dimensional rigid-body collisions with friction, J. Appl. Mech. 59 (1992) 635.

[19] The eddy current separator used for the experiments is model BM $29.701 / 18$, from Bakker Magnetics, Son, The Netherlands. 